Bull. Korean Math. Soc. 47 (2010), No. 4, pp. 693-699

DOI 10.4134/BKMS.2010.47.4.693

\title{
INVERSE POLYNOMIAL MODULES INDUCED BY AN R-LINEAR MAP
}

\author{
SAngwon Park And Jinsun JeOng
}

\begin{abstract}
In this paper we show that the flat property of a left $R$ module does not imply (carry over) to the corresponding inverse polynomial module. Then we define an induced inverse polynomial module as an $R[x]$-module, i.e., given an $R$-linear map $f: M \rightarrow N$ of left $R$-modules, we define $N+x^{-1} M\left[x^{-1}\right]$ as a left $R[x]$-module. Given an exact sequence of left $R$-modules

$$
0 \longrightarrow N \longrightarrow E^{0} \longrightarrow E^{1} \longrightarrow 0
$$

where $E^{0}, E^{1}$ injective, we show $E^{1}+x^{-1} E^{0}\left[\left[x^{-1}\right]\right]$ is not an injective left $R[x]$-module, while $E^{0}\left[\left[x^{-1}\right]\right]$ is an injective left $R[x]$-module. Make a left $R$-module $\mathrm{N}$ as a left $R[x]$-module by $x N=0$. We show

$$
\operatorname{inj} \operatorname{dim}_{R} N=n \quad \text { implies } \quad \operatorname{inj} \operatorname{dim}_{R[x]} N=n+1
$$
\end{abstract}

by using the induced inverse polynomial modules and their properties.

\section{Introduction}

If $R$ is a left Noetherian ring, then for an injective left $R$-module $E, E\left[x^{-1}\right]$ is an injective left $R[x]$-module $([2],[3])$. But for a projective left $R$-module $P, P\left[x^{-1}\right]$ is not a projective left $R[x]$-module, in general ([5]). We extend this question to the flat module and we show that for a flat left $R$-module $F$, $F\left[x^{-1}\right]$ is not a flat left $R[x]$-module, in general. Then we construct an induced inverse polynomial as an $R[x]$-module. Let $M$ and $N$ be left $R$-modules and $f: M \rightarrow N$ be an $R$-linear map. Then we can define $N+x^{-1} M\left[x^{-1}\right]$ as a left $R[x]$-module defined by

$$
x\left(b_{0}+a_{1} x^{-1}+\cdots+a_{n} x^{-n}\right)=b_{1}+a_{2} x^{-1}+\cdots+a_{n} x^{-n+1},
$$

where $f\left(a_{1}\right)=b_{1}, b_{0} \in N$, and $a_{i} \in M$. Given an exact sequence of $R$-modules

$$
0 \longrightarrow N \longrightarrow E^{0} \longrightarrow E^{1} \longrightarrow 0,
$$

Received January 29, 2009.

2000 Mathematics Subject Classification. Primary 16E30; Secondary 13C11, 16D80.

Key words and phrases. flat module, injective module, inverse polynomial module, induced module.

This study was supported by research funds from Dong-A University. 
where $E^{0}, E^{1}$ are injective, we show $E^{1}+x^{-1} E^{0}\left[\left[x^{-1}\right]\right]$ is not an injective left $R[x]$-module, while $E^{0}\left[\left[x^{-1}\right]\right]$ is an injective left $R[x]$-module. Make a left $R$-module $N$ as a left $R[x]$-module by $x N=0$. We show

$$
\operatorname{inj} \operatorname{dim}_{R} N=n \quad \text { implies } \quad \operatorname{inj} \operatorname{dim}_{R[x]} N=n+1
$$

by using the inverse polynomial modules. Inverse polynomial modules were developed in ([1], [6], [7], [8]) recently.

Definition 1.1 ([4]). Let $R$ be a ring and $M$ be a left $R$-module. Then $M\left[x^{-1}\right]$ is a left $R[x]$-module defined by

$$
x\left(m_{0}+m_{1} x^{-1}+\cdots+m_{i} x^{-i}\right)=m_{1}+m_{2} x^{-1}+\cdots+m_{i} x^{-i+1}
$$

and such that

$$
r\left(m_{0}+m_{1} x^{-1}+\cdots+m_{n} x^{-n}\right)=r m_{0}+r m_{1} x^{-1}+\cdots+r m_{n} x^{-n},
$$

where $r \in R$. We call $M\left[x^{-1}\right]$ as an inverse polynomial module.

Similarly, we can define $\left.M\left[\left[x^{-1}\right]\right], M\left[x, x^{-1}\right], M\left[\left[x, x^{-1}\right]\right], M\left[x, x^{-1}\right]\right]$ and $M\left[\left[x, x^{-1}\right]\right.$ as left $R[x]$-modules where, for example, $M\left[\left[x, x^{-1}\right]\right.$ is the set of Laurent series in $x$ with coefficients in $M$, i.e., the set of all formal sums $\sum_{k \geq n_{0}} m_{k} x^{k}$ with $n_{0}$ any element of $\mathbb{Z}$ ( $\mathbb{Z}$ is the set of all integers).

Lemma $1.2([8])$. Let $E$ be a left $R$-module. Then $E\left[\left[x^{-1}\right]\right]$ is an injective left $R[x]$-module.

Lemma 1.3. If $E\left[\left[x^{-1}\right]\right]$ is an injective left $R[x]$-module, then $E$ is an injective left $R$-module.

Proof. Let $I$ be a left ideal of $R$ and $f: I \rightarrow E$ be an $R$-linear map. Then since $E\left[\left[x^{-1}\right]\right]$ is an injective left $R[x]$-module, we can complete the following diagram by $g$

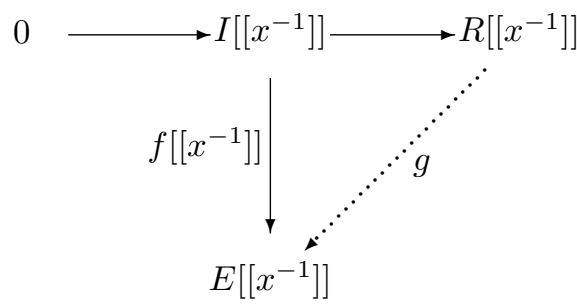

as a commutative diagram, where $f\left[\left[x^{-1}\right]\right]\left(\sum_{i=0}^{\infty} r_{i} x^{-i}\right)=\sum_{i=0}^{\infty} f\left(r_{i}\right) x^{-i}$. Since $x R=0, x g(R)=0$ in $E\left[\left[x^{-1}\right]\right]$. But this implies $g(R) \subset E$. Thus ${ }_{E}|g|_{R}: R \rightarrow E$ can complete the following diagram 


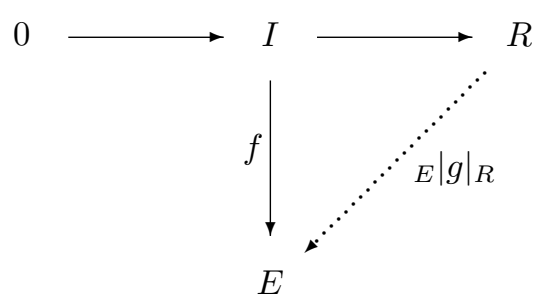

as a commutative diagram. Hence, $E$ is an injective left $R$-module.

Lemma 1.4. Let $M$ be a left R-module. Then

$$
\operatorname{inj} \operatorname{dim}_{R[x]} M\left[\left[x^{-1}\right]\right]=\operatorname{inj} \operatorname{dim}_{R} M .
$$

Proof. Suppose $\operatorname{inj} \operatorname{dim}_{R} M=n$ and

$$
0 \rightarrow M \rightarrow E^{0} \rightarrow E^{1} \rightarrow \cdots \rightarrow E^{n} \rightarrow 0
$$

is an injective resolution of $M$. Then by Lemma 1.2 , for each $i, E^{i}\left[\left[x^{-1}\right]\right]$ is an injective left $R[x]$-module and

$$
0 \rightarrow M\left[\left[x^{-1}\right]\right] \rightarrow E^{0}\left[\left[x^{-1}\right]\right] \rightarrow E^{1}\left[\left[x^{-1}\right]\right] \rightarrow \cdots \rightarrow E^{n}\left[\left[x^{-1}\right]\right] \rightarrow 0
$$

is an injective resolution of $M\left[\left[x^{-1}\right]\right]$. Let $K^{i}=\operatorname{Ker}\left(E^{i} \rightarrow E^{i+1}\right)$ for $0 \leq$ $i<n$. Then $K^{i}$ is not an injective left $R$-module for $0 \leq i<n$. So by Lemma $1.3, K^{i}\left[\left[x^{-1}\right]\right]$ is not an injective left $R[x]$-module. So then we get $\operatorname{inj} \operatorname{dim}_{R[x]} M\left[\left[x^{-1}\right]\right]=n$. Suppose $\operatorname{inj} \operatorname{dim}_{R} M=\infty$ and

$$
0 \rightarrow M \rightarrow E^{0} \rightarrow E^{1} \rightarrow \cdots \rightarrow E^{n} \rightarrow \cdots
$$

is an injective resolution of $M$. Then

$$
0 \rightarrow M\left[\left[x^{-1}\right]\right] \rightarrow E^{0}\left[\left[x^{-1}\right]\right] \rightarrow E^{1}\left[\left[x^{-1}\right]\right] \rightarrow \cdots \rightarrow E^{n}\left[\left[x^{-1}\right]\right] \rightarrow \cdots
$$

is an injective resolution of $M\left[\left[x^{-1}\right]\right]$. But $K^{i}$ is not an injective left $R$-module for all $i$. Thus $K^{i}\left[\left[x^{-1}\right]\right]$ is not an injective left $R[x]$-module for all $i$. Therefore, $\operatorname{inj} \operatorname{dim}_{R[x]} M\left[\left[x^{-1}\right]\right]=\infty$. Similarly, if inj $\operatorname{dim}_{R[x]} M\left[\left[x^{-1}\right]\right]=n$, then $\operatorname{inj} \operatorname{dim}_{R} M=n$, and if inj $\operatorname{dim}_{R[x]} M\left[\left[x^{-1}\right]\right]=\infty$, then inj $\operatorname{dim}_{R} M=\infty$. Hence, $\operatorname{inj} \operatorname{dim}_{R[x]} M\left[\left[x^{-1}\right]\right]=\operatorname{inj} \operatorname{dim}_{R} M$.

\section{Flat module}

Lemma 2.1. Let $M$ be a left $R$-module. Then $R[x] \otimes_{R[x]} M\left[x^{-1}\right] \cong M\left[x^{-1}\right]$.

Proof. Define $\phi: M\left[x^{-1}\right] \rightarrow R[x] \otimes M\left[x^{-1}\right]$ by $\phi(f)=1 \otimes f$ and $\psi: R[x] \otimes$ $M\left[x^{-1}\right] \rightarrow M\left[x^{-1}\right]$ by $\psi(x \otimes f)=x f$. Then $\phi$ and $\psi$ are $R[x]$-linear maps. And

$$
\begin{gathered}
(\phi \circ \psi)(x \otimes f)=\phi(\psi(x \otimes f))=\phi(x f)=1 \otimes x f=x \otimes f, \\
(\psi \circ \phi)(f)=\psi(\phi(f))=\psi(1 \otimes f)=f .
\end{gathered}
$$

Hence, $R[x] \otimes_{R[x]} M\left[x^{-1}\right] \cong M\left[x^{-1}\right]$. 
Similarly, we can get $R[x] \otimes_{R[x]} M\left[\left[x^{-1}\right]\right] \cong M\left[\left[x^{-1}\right]\right]$.

Theorem 2.2. If $F$ is a flat left $R$-module, then $F\left[x^{-1}\right]$ is not a flat left $R[x]$-module, in general.

Proof. Let $R=\mathbb{R}$ (the ring of real numbers). Let $\phi: R[x] \rightarrow R[x]$ by $\phi(f)=$ $x f$. Then $\phi$ is an injective $R[x]$-linear map. Then $\phi \otimes_{\mathbb{R}[x]} \operatorname{id}_{F\left[x^{-1}\right]}: \mathbb{R}[x] \otimes_{\mathbb{R}[x]}$ $F\left[x^{-1}\right] \rightarrow \mathbb{R}[x] \otimes_{\mathbb{R}[x]} F\left[x^{-1}\right]$ is defined by $\phi \otimes_{\mathbb{R}[x]} \operatorname{id}_{F\left[x^{-1}\right]}\left(a x \otimes b x^{-1}\right)=a x^{2} \otimes$ $b x^{-1}$, where $a, b \in \mathbb{R}$. Since $\mathbb{R}[x] \otimes_{\mathbb{R}[x]} F\left[x^{-1}\right] \cong F\left[x^{-1}\right]$, we have the following

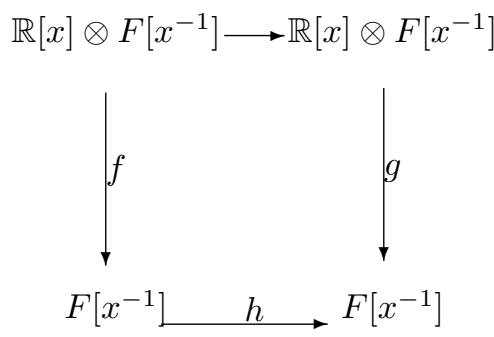

commutative diagram. But $(h \circ f)\left(a x \otimes b x^{-1}\right)=\left(g \circ \phi \otimes_{\mathbb{R}[x]} \operatorname{id}_{F\left[x^{-1}\right]}\right)\left(a x \otimes b x^{-1}\right)$ implies $h(a b)=0$. Thus $h: F\left[x^{-1}\right] \rightarrow F\left[x^{-1}\right]$ is not injective, so that $\phi \otimes_{\mathbb{R}[x]}$ $\operatorname{id}_{F\left[x^{-1}\right]}$ is not injective. Hence, $F\left[x^{-1}\right]$ is not a flat left $R[x]$-module.

Remark 1. Since $R[x] \otimes_{R[x]} M\left[\left[x^{-1}\right]\right] \cong M\left[\left[x^{-1}\right]\right]$, we also see that $F\left[\left[x^{-1}\right]\right]$ is not a flat left $R[x]$-module.

\section{Induced inverse polynomial modules}

Definition 3.1. Let $f: M \rightarrow N$ be an $R$-linear map. Then $N+x^{-1} M\left[x^{-1}\right]$ is a left $R[x]$-module defined by

$$
x\left(b_{0}+a_{1} x^{-1}+\cdots+a_{n} x^{-n}\right)=b_{1}+a_{2} x^{-1}+\cdots+a_{n} x^{-n+1},
$$

where $f\left(a_{1}\right)=b_{1}, b_{0} \in N, a_{i} \in M$.

Similarly, we can define $N+x^{-1} M\left[\left[x^{-1}\right]\right]$ as a left $R[x]$-module.

Note. Given a left $R$-module $M$, we can make $M$ as a left $R[x]$-module by defining $x M=0$.

Lemma 3.2. If $0 \rightarrow L \stackrel{f}{\longrightarrow} M \stackrel{g}{\longrightarrow} N \rightarrow 0$ is a short exact sequence of $R$ modules, then

$$
0 \rightarrow L \rightarrow M\left[x^{-1}\right] \rightarrow N+x^{-1} M\left[x^{-1}\right] \rightarrow 0
$$

is a short exact sequence of $R[x]$-modules. 
Proof. Let $f\left[x^{-1}\right]: L \rightarrow M\left[x^{-1}\right]$ be defined by $f\left[x^{-1}\right](n)=f(n)$ for $n \in L$. Then since $f$ is an injective $R$-linear map, $f\left[x^{-1}\right]$ is an injective $R[x]$-linear map. Let $g\left[x^{-1}\right]: M\left[x^{-1}\right] \rightarrow N+x^{-1} M\left[x^{-1}\right]$ be defined by

$g\left[x^{-1}\right]\left(e_{0}+e_{1} x^{-1}+e_{2} x^{-2}+\cdots+e_{i} x^{-i}\right)=g\left(e_{0}\right)+e_{1} x^{-1}+e_{2} x^{-2}+\cdots+e_{i} x^{-i}$.

Then easily $g\left[x^{-1}\right]$ is an $R[x]$-linear map. Let $b_{0}+e_{1} x^{-1}+e_{2} x^{-2}+\cdots+e_{i} x^{-i} \in$ $N+x^{-1} M\left[x^{-1}\right]$. Then since $g$ is a surjective $R$-linear map, there exists $e_{0} \in M$ such that $g\left(e_{0}\right)=b_{0}$. So, $g\left[x^{-1}\right]$ is a surjective $R[x]$-linear map. Now

$$
\begin{aligned}
\left(g\left[x^{-1}\right] \circ f\left[x^{-1}\right]\right)(n) & =g\left[x^{-1}\right](f(n)) \\
& =g(f(n)) \\
& =0 .
\end{aligned}
$$

And if $e_{0}+e_{1} x^{-1}+e_{2} x^{-2}+\cdots+e_{i} x^{-i} \in \operatorname{Ker} g\left[x^{-1}\right]$, where $e_{i} \in M$, then

$$
\begin{aligned}
& g\left[x^{-1}\right]\left(e_{0}+e_{1} x^{-1}+e_{2} x^{-2}+\cdots+e_{i} x^{-i}\right) \\
= & g\left(e_{0}\right)+e_{1} x^{-1}+e_{2} x^{-2}+\cdots+e_{i} x^{-i} \\
= & 0 .
\end{aligned}
$$

So $g\left(e_{0}\right)=0, e_{1}=e_{2}=\cdots=e_{i}=0$, which implies $e_{0} \in \operatorname{Ker} g=\operatorname{Im} f=f(L)$.

Hence,

$$
0 \rightarrow L \rightarrow M\left[x^{-1}\right] \rightarrow N+x^{-1} M\left[x^{-1}\right] \rightarrow 0
$$

is a short exact sequence of $R[x]$-modules.

Similarly, given a short exact sequence $0 \rightarrow L \rightarrow M \rightarrow N \rightarrow 0$ of $R$-modules, we get a short exact sequence $0 \rightarrow L \rightarrow M\left[\left[x^{-1}\right]\right] \rightarrow N+x^{-1} M\left[\left[x^{-1}\right]\right] \rightarrow 0$ of $R[x]$-modules.

Lemma 3.3. Let $0 \rightarrow N \stackrel{f}{\longrightarrow} E^{0} \stackrel{g}{\longrightarrow} E^{1} \rightarrow 0$ be a short exact sequence of $R$-modules, where $E^{0}, E^{1}$ are injective with inj $\operatorname{dim}_{R} N=1$. Then $E^{1}+$ $x^{-1} E^{0}\left[\left[x^{-1}\right]\right]$ is not an injective left $R[x]$-module.

Proof. Suppose $E^{1}+x^{-1} E^{0}\left[\left[x^{-1}\right]\right]$ is an injective left $R[x]$-module. Then there exists a $R[x]$-linear map $\phi$ which completes the following diagram

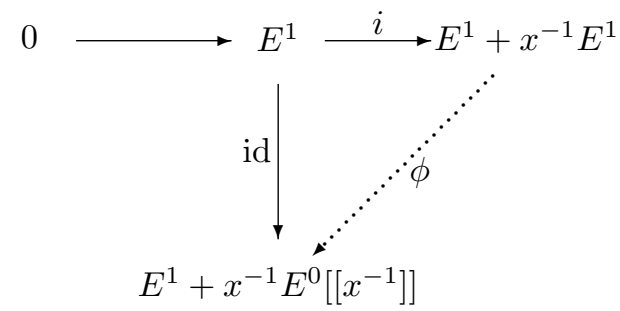


as a commutative diagram.

Then there exists an $R$-linear map $h: E^{1} \rightarrow E^{0}$ such that $g \circ h=\operatorname{id}_{E^{1}}$. But since $\operatorname{inj} \operatorname{dim}_{R} N=1,0 \rightarrow N \stackrel{f}{\longrightarrow} E^{0} \stackrel{g}{\longrightarrow} E^{1} \rightarrow 0$ is not split, which implies a contradiction. Hence, $E^{1}+x^{-1} E^{0}\left[\left[x^{-1}\right]\right]$ is not an injective left $R[x]$ module.

Similarly, given a short exact sequence $0 \rightarrow N \rightarrow E^{0} \rightarrow E^{1} \rightarrow 0$ of $R$-modules with $E^{0}, E^{1}$ injective and $i n j \operatorname{dim}_{R} N=1$, we see that $E^{1}+$ $x^{-1} E^{0}\left[x^{-1}\right]$ is not an injective left $R[x]$-module.

Theorem 3.4. Let $\operatorname{inj} \operatorname{dim}_{R} N=n($ with $N \neq 0)$. Make $N$ into an left $R[x]$ module so that $x N=0$. Then

$$
\operatorname{inj} \operatorname{dim}_{R[x]} N=n+1 .
$$

Proof. Let $N$ be a left $R$-module. Then

$$
\operatorname{inj} \operatorname{dim}_{R} N=\operatorname{inj} \operatorname{dim}_{R[x]} N\left[\left[x^{-1}\right]\right]=n .
$$

And we have the short exact sequence of $R[x]$-modules

$$
0 \rightarrow N \rightarrow N\left[\left[x^{-1}\right]\right] \rightarrow N\left[\left[x^{-1}\right]\right] \rightarrow 0 .
$$

Then $\operatorname{inj} \operatorname{dim}_{R[x]} N \leq\left(\operatorname{inj} \operatorname{dim}_{R} N\right)+1=n+1$. Since if $N$ is an injective $R[x]$-module, then $N$ is an injective $R$-module so that

$$
\operatorname{inj} \operatorname{dim}_{R} N \leq \operatorname{inj} \operatorname{dim}_{R[x]} N \leq\left(\operatorname{inj} \operatorname{dim}_{R} N\right)+1 .
$$

Now by induction on $n$, if $n=0$, then we want to show $\operatorname{inj} \operatorname{dim}_{R[x]} N=1$. But inj $\operatorname{dim}_{R} N=0$ means that $N$ is an injective $R$-module. If $N$ is an injective $R[x]$-module, then $N$ is divisible by $x$. But $x N=0$. Thus $N$ is not divisible by $x$. Thus $N$ is not an injective $R[x]$-module. Therefore, inj $\operatorname{dim}_{R[x]} N \neq 0$, i.e., $\operatorname{inj} \operatorname{dim}_{R[x]} N=1$.

If $n=1$, then we have a short exact sequence $0 \rightarrow N \rightarrow E^{0} \rightarrow E^{1} \rightarrow 0$ of $R$-modules with $E^{0}, E^{1}$ injective. Then by Lemma $3.3, E^{1}+x^{-1} E^{0}\left[\left[x^{-1}\right]\right]$ is not an injective left $R[x]$-module and by Lemma $3.2,0 \rightarrow N \rightarrow E^{0}\left[\left[x^{-1}\right]\right] \rightarrow$ $E^{1}+x^{-1} E^{0}\left[\left[x^{-1}\right]\right] \rightarrow 0$ is a short exact sequence. Therefore, inj $\operatorname{dim}_{R[x]} N=2$.

Now we suppose $\operatorname{inj} \operatorname{dim}_{R} N=n>1$ and make the obvious induction hypothesis. Let $0 \rightarrow N \rightarrow E \rightarrow L \rightarrow 0$ be an exact sequence of left $R$ modules with $E$ injective. Then $\operatorname{inj} \operatorname{dim}_{R} L=n-1$. Now make $N, E, L$ into $R[x]$-modules with $x N=0, x E=0, x L=0$. Then inj $\operatorname{dim}_{R[x]} E=1$ and by the induction hypothesis we know inj $\operatorname{dim}_{R[x]} L=n$. Using the long exact sequence of $\operatorname{Ext}_{R[x]}(A,-)$ where $A$ is any left $R$-module, we get that inj $\operatorname{dim}_{R[x]} N=n+1$.

\section{References}

[1] Z. Liu, Injectivity of modules of generalized inverse polynomials, Comm. Algebra 29 (2001), no. 2, 583-592.

[2] A. S. McKerrow, On the injective dimension of modules of power series, Quart. J. Math. Oxford Ser. (2) 25 (1974), 359-368. 
[3] D. G. Northcott, Injective envelopes and inverse polynomials, J. London Math. Soc. (2) 8 (1974), 290-296.

[4] S. Park, Inverse polynomials and injective covers, Comm. Algebra 21 (1993), no. 12, 4599-4613.

[5] _ The Macaulay-Northcott functor, Arch. Math. (Basel) 63 (1994), no. 3, 225-230.

[6] _ Gorenstein rings and inverse polynomials, Comm. Algebra 28 (2000), no. 2, $785-789$.

[7] _ The general structure of inverse polynomial modules, Czechoslovak Math. J. 51(126) (2001), no. 2, 343-349.

[8] S. Park and E. Cho, Injective and projective properties of $R[x]$-modules, Czechoslovak Math. J. 54(129) (2004), no. 3, 573-578.

SANGWON PARK

Department of Mathematics

DONG-A University

Pusan 604-714, Korea

E-mail address: swpark@donga.ac.kr

JINSUN JEONG

Department of Mathematics

Dong-A University

PUSAN 604-714, Korea

E-mail address: jsjeong@donga.ac.kr 